\title{
Effectiveness of Current Treatments for Wet Age-Related Macular Degeneration in Japan: A Systematic Review and Pooled Data Analysis
}

\author{
Kanji Takahashi ${ }^{1}$, Tomohiro lida ${ }^{2}$, Susumu Ishida ${ }^{3}$, Bruce Crawford ${ }^{4}$, Yoko Sakai ${ }^{4}$, \\ Akikazu Mochizuki $\mathbb{D D}^{5}$, Ryuta Tsujiuchi ${ }^{5}$, Satoru Tanaka ${ }^{5}$, Kota Imai ${ }^{5}$ \\ 'Department of Ophthalmology, Kansai Medical University, Hirakata, Osaka, Japan; ${ }^{2}$ Department of Ophthalmology, Tokyo Women's Medical \\ University, Shinjuku, Tokyo, Japan; ${ }^{3}$ Department of Ophthalmology, Graduate School of Medicine, Hokkaido University, Sapporo, Hokkaido, Japan; \\ ${ }^{4}$ Syneos Health Clinical K.K., Chuo-ku, Tokyo, Japan; ${ }^{5}$ Novartis Pharma K.K., Minato-ku, Tokyo, Japan \\ Correspondence: Akikazu Mochizuki, Novartis Pharma K.K., 23-I, Toranomon I-chome, Minato-ku, Tokyo, Japan, Tel +8I 3 6899 8000, \\ Fax +8I 36257 3634, Email akikazu.mochizuki@novartis.com
}

\begin{abstract}
Purpose: We conducted a systematic review to investigate the effectiveness of clinical treatments for wet age-related macular degeneration (wAMD) in Japanese patients in the decade since anti-vascular endothelial growth factor (VEGF) therapies were introduced.

Methods: PubMed was searched for articles published in English between 1 January 2008 and 30 September 2018 using a multistring search strategy. Reviews were scanned for additional relevant studies and select gray literature was evaluated. Mean and/or median for the logarithm of the minimum angle of resolution (logMAR) visual acuity (VA), central retinal thickness (CRT), and the number of injections after 12 months of treatment were calculated using extracted data. Data were stratified by disease type and treatment modality.

Results: Of 335 studies identified, 94 were selected for data extraction (147 treatment arms; typical AMD, n = 25; polypoidal choroidal vasculopathy [PCV], $\mathrm{n}=85)$. Mean (median) logMAR VA was $0.44(0.32)$ for typical AMD and $0.34(0.31)$ for PCV; the respective mean number of anti-VEGF injections was 5.6 and 4.6. The mean CRT was approximately $220 \mu \mathrm{m}$ for both groups. For typical AMD, anti-VEGF monotherapy resulted in better VA outcomes than photodynamic therapy (PDT) alone. For PCV, anti-VEGF monotherapy or anti-VEGF plus PDT combination therapy resulted in better VA and CRT outcomes than PDT monotherapy. Combination therapy required fewer injections than anti-VEGF monotherapy (PCV, 3.2 versus 5.3).

Conclusion: wAMD treatment has advanced dramatically in the years since anti-VEGF drugs were introduced in Japan. Discrete patient populations may benefit from differing management regimens, including the fewer injections required with combination therapy.
\end{abstract}

Keywords: visual acuity, polypoidal choroidal vasculopathy, anti-vascular endothelial growth factor therapy, photodynamic therapy

\section{Introduction}

Globally, age-related macular degeneration (AMD) is the third leading cause of blindness following cataracts and glaucoma ${ }^{1}$ and is the primary cause of blindness in industrialized countries. ${ }^{2}$ AMD results from changes in the macular region of the retina in older people (aged $>55$ years), affecting photoreceptors and the retinal pigment epithelium, leading to a progressive loss of vision. ${ }^{3}$ The incidence of AMD is increasing as the global population ages, ${ }^{4,5}$ and management of age-related diseases over the coming decades is of particular concern in countries with a large proportion of elderly citizens, such as Japan. Population-based cohort studies in Japan have indicated that the overall prevalence of AMD ranges from $13.4 \%{ }^{6}$ to $21.5 \%,{ }^{7}$ with a notable increase between the ages of $50-59$ years $(16.4 \%)$ and $70-74$ years (32.2\%). ${ }^{8}$ Furthermore, the number of Japanese patients with AMD is expected to increase over the next 1-2 decades, potentially peaking in $2035 .{ }^{9}$ 
Wet AMD (wAMD), also known as exudative or neovascular AMD, develops in about $10 \%$ of all AMD cases ${ }^{10}$ and arises due to blood vessels from the choroid growing into the normally avascular retinal pigment epithelium and subretinal regions. ${ }^{11}$ wAMD is an infrequent complication of AMD, with an estimated global prevalence rate of $0.46 \%{ }^{4}$ However, wAMD accounts for $80-90 \%$ of the severe vision loss associated with AMD. ${ }^{12}$ Furthermore, one phenotype of wAMD called polypoidal choroidal vasculopathy (PCV) is known to occur at a higher prevalence in Japanese and Asian individuals than Caucasians. ${ }^{13-15}$ Patients with wAMD rapidly lose the central vision required for tasks such as reading, screen use, and driving, ${ }^{16}$ thus restricting both leisure activities and the ability to work, and severely reducing quality of life. ${ }^{16-18}$ The economic burden caused by visual impairment is substantial, in Japan; in 2007, it was estimated to be about 8785 billion yen, which equated to $1.7 \%$ of the gross domestic product in that year. ${ }^{19}$

The treatment algorithm for wAMD in Japan is driven by disease severity, and the recommended standard-of-care is anti-vascular endothelial growth factor (anti-VEGF) therapy. ${ }^{20}$ This class of drug binds to excess VEGF protein that would otherwise promote the erroneous growth of new blood vessels. ${ }^{21}$ As this protein is also essential for normal tissue healing and growth, VEGF must be only suppressed in the eye; therefore, these drugs are administered via intraocular injections on an intermittent or pro re nata (PRN) schedule. ${ }^{21}$ Anti-VEGF inhibitors first became available in Japan in $2008,{ }^{22}$ and drugs in this class now approved in Japan include the monoclonal antibody ranibizumab and the synthetic protein aflibercept, both of which have proven clinical effectiveness in wAMD. ${ }^{23-27}$ To treat wAMD in Japan, antiVEGF drugs are used either as monotherapy or in combination with photodynamic therapy (PDT) ${ }^{20}$

Drug development for wAMD is ongoing, ${ }^{28-30}$ and as new therapies become available, it is vital that Japanese patients and clinicians have the necessary information to assess management options critically. This, in turn, will allow them to make optimal treatment decisions. Thus, the primary objective of this study was to systematically review randomized controlled trials, observational studies, case studies, and treatment guidelines to investigate the effectiveness of clinical treatments for wAMD in Japanese patients in the decade following the 2008 introduction of anti-VEGF therapies.

\section{Methods}

\section{Data Sources and Search Methodology}

The methodology used for this systematic review was guided by the Preferred Reporting Items for Systematic Reviews and Meta-Analyses (PRISMA) guidelines ${ }^{31}$ (S1 Text) and documentation from the National Institute for Health and Clinical Excellence in the United Kingdom. ${ }^{32}$ The protocol was not registered. A comprehensive search was conducted in PubMed for articles published in English between 1 January 2008 and 30 September 2018. A multistring search strategy was implemented, and either title/abstract (TIAB) or medical subject heading (MeSH) entries were searched ( $\underline{\mathrm{S} 2 \mathrm{Text}}$ ).

\section{Study Selection Criteria}

In the first round of screening, titles and abstracts of all records retrieved from the literature search were reviewed by YS and William YuanHao Kuan (Syneos Health, Tokyo, Japan). The relevance of each article was evaluated based on the inclusion and exclusion criteria (Table 1). A Population, Intervention, Comparison, and Outcome strategy was used to construct the specific research question. The AMD diagnostic criteria specified were those published by the Japanese Ministry of Health, Labour and Welfare. ${ }^{33}$ Studies of patients with retinal angiomatous proliferation were excluded, as this is a unique type of wAMD. Additional inclusion and exclusion criteria included the type of article, sample size, and geographic setting. Citations that did not match the eligibility criteria and duplicate citations were excluded. Unclear citations were included for more detailed evaluation. Any disparities were resolved by discussion between all authors. After reaching a consensus, the full text of studies identified as potentially relevant was reviewed in a second screening round and included in the analyses if the studies fulfilled the specified criteria.

\section{Data Extraction}

The data presented in the selected studies were extracted by William YuanHao Kuan (Syneos Health), and the quality was checked by YS. The extracted information included type of study or article, number of sites, study duration, number 
Table I Inclusion and Exclusion Criteria for the First Round of Literature Evaluation

\begin{tabular}{|c|c|c|}
\hline Item & Inclusion & Exclusion \\
\hline \multicolumn{3}{|l|}{ PICO strategy } \\
\hline Patient population & $\begin{array}{l}\text { Japanese patients aged } \geq 50 \text { years with choroidal } \\
\text { neovascularization who meet Japanese wAMD } \\
\text { diagnostic criteria }^{29}\end{array}$ & $\begin{array}{l}\text { Patients with central serous chorioretinopathy, lesions from } \\
\text { myopia, and other ocular diseases causing vision impairment, } \\
\text { including inflammatory and degenerative diseases }\end{array}$ \\
\hline Intervention/comparator & $\begin{array}{l}\text { Any approved, widely-used, or guideline- } \\
\text { recommended interventional therapy in Japan }{ }^{\mathrm{a}, 19}\end{array}$ & $\begin{array}{l}\text { Studies not reporting any of the listed interventions/ } \\
\text { comparators of interest }\end{array}$ \\
\hline Outcomes & $\begin{array}{l}\text { Reporting of VA, central retinal thickness, and/or } \\
\text { number of injections at } 12 \text { months }\end{array}$ & Studies not reporting outcomes of interest \\
\hline \multicolumn{3}{|l|}{ Other criteria } \\
\hline Article type & $\begin{array}{l}\text { RCT, observational study, case series, } \\
\text { conference proceeding, current wAMD } \\
\text { diagnosis/treatment guidelines }\end{array}$ & Non-systematically conducted reviews or editorials \\
\hline Sample size & $\geq 10$ Japanese patients with a diagnosis of wAMD & $<10$ patients \\
\hline Geographic setting & Japan & Any other country \\
\hline
\end{tabular}

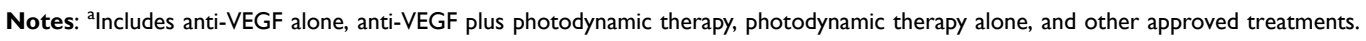

Abbreviations: PICO, Population, Intervention, Comparison, and Outcome; RCT, randomized controlled trial; VA, visual acuity; VEGF, vascular endothelial growth factor; wAMD, wet age-related macular degeneration.

of patients, baseline demographic and clinical characteristics, type of wAMD, risk factors, details of diagnosis and management, and clinical efficacy endpoints after 1 year of treatment (including visual acuity [VA], central retinal thickness [CRT], and number of injections [if applicable]). For dichotomous outcomes, the number of patients analyzed $(\mathrm{N})$ and the number of patients with the outcome (n) were extracted. For continuous outcomes, N, mean, median, and interquartile range (IQR) were recorded.

\section{Statistical Methods}

Data from all relevant studies were pooled to report mean and/or median (IQR) for the logarithm of the minimum angle of resolution (logMAR) VA, CRT, and the number of injections. Data were stratified by type of wAMD and/or type of treatment. To explore the clinical efficacy endpoints stratified by these factors, Box and Whisker plots were created (indicating the median, 25th, and 75th percentile values). No adjustment was made for the data quantity (number of articles/treatment arms) or variations in baseline parameters. Data reported using the Early Treatment Diabetic Retinopathy Study scale was not converted into $\operatorname{logMAR}$. As we did not adjust the data weighting of the papers included in the analysis, no tests for statistical difference were conducted. Microsoft Excel 2016 was used for all calculations.

\section{Results}

\section{Article Selection}

A PRISMA flowchart of the identification process is shown in Figure 1. The initial search identified 335 studies. After removing duplicates and those articles that did not meet the eligibility criteria, 280 articles remained for the detailed second-round assessment. An additional 17 gray literature articles were also subjected to detailed evaluation. Articles were excluded based on the patient population and study design, then by intervention, and finally by outcome. Following the full-text evaluation, 94 articles were selected for data extraction, resulting in a total of 147 individual treatment arms with available data. A full list of the 94 selected articles is provided as S1 Table. 


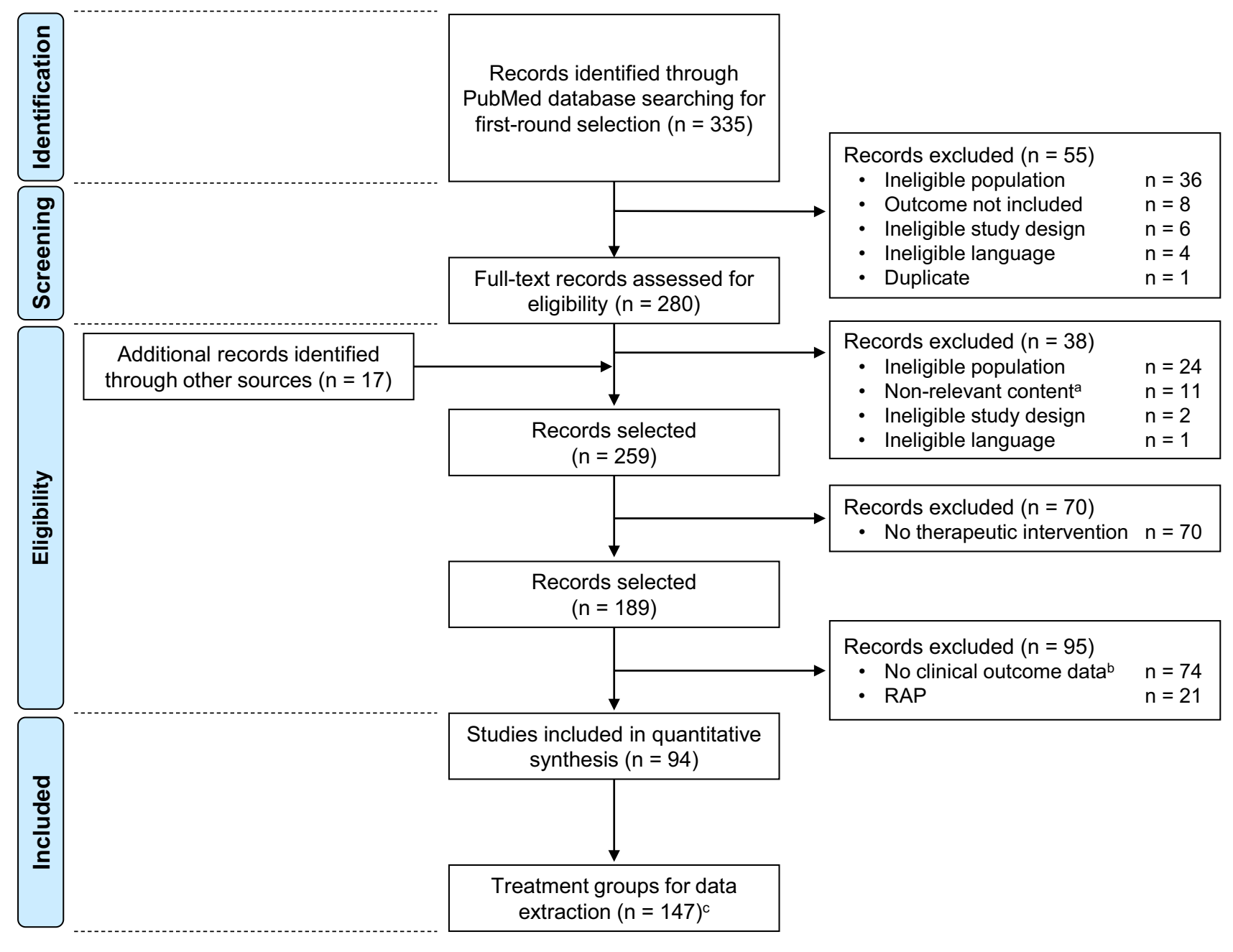

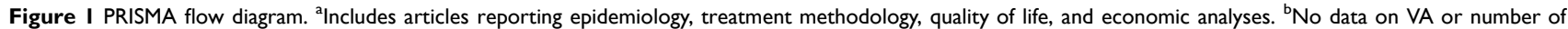
injections after 12 months of treatment. ${ }^{\mathrm{C}}$ Total number of individual treatment arms for which data were reported.

Abbreviations: PRISMA, Preferred Reporting Items for Systematic reviews and Meta-Analyses; RAP, retinal angiomatous proliferation; VA, visual acuity.

\section{Stratification Factors}

A breakdown of the disease types and treatment modalities in the 147 treatment groups is shown in Table 2. More treatment arms included patients with PCV $(n=85)$ than with typical AMD $(n=25)$. The two main types of treatments for wAMD were anti-VEGF injections (generally intravitreal aflibercept or intravitreal ranibizumab) and PDT, either as

Table 2 Breakdown of Data Stratification Factors of the 147 Evaluable Treatment Groups

\begin{tabular}{|c|c|c|c|c|c|c|}
\hline & & \multicolumn{5}{|c|}{ Treatment Modality } \\
\hline & & Anti-VEGF & Anti-VEGF + PDT & PDT & Other ${ }^{a}$ & Total \\
\hline \multirow[t]{4}{*}{ Type of wAMD } & Typical AMD & 20 & 1 & 3 & I & 25 \\
\hline & PCV & 40 & 23 & 19 & 3 & 85 \\
\hline & Other $^{b}$ & 24 & 2 & 5 & 6 & 37 \\
\hline & Total & 84 & 26 & 27 & 10 & 147 \\
\hline
\end{tabular}

Notes: ${ }^{a}$ Includes treatment with steroids or pegaptanib. ${ }^{b}$ Disease type that cannot be classified as typical AMD or PCV.

Abbreviations: AMD, age-related macular degeneration; PCV, polypoidal choroidal vasculopathy; PDT, photodynamic therapy; VEGF, vascular endothelial growth factor; wAMD, wet AMD 
monotherapy or in combination. Interventions such as bevacizumab, pegaptanib, brolucizumab, and triamcinolone acetonide were rarely reported. A visualization of the treatment arms included in each outcome calculation is shown

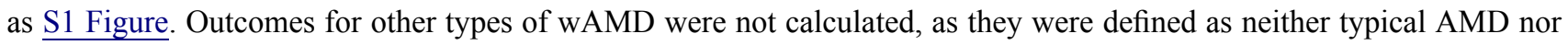
PCV. Additionally, outcomes were not calculated for other types of treatment because they included off-label steroid treatment and pegaptanib, which is rarely used in clinical practice.

\section{Outcomes}

Data for VA after 12 months of treatment, stratified by disease type, are shown in Figure 2A. The mean (median) $\log$ MAR VA after 12 months of treatment was 0.44 (0.32) for typical AMD and $0.34(0.31)$ for PCV.

Data for VA after 12 months of treatment, stratified by disease type and treatment modality, are shown in Figure 2B-C. For typical AMD, VA after 12 months of treatment tended to be better with anti-VEGF monotherapy than with PDT monotherapy. For PCV, post-treatment VA tended to be better with anti-VEGF monotherapy or with anti-VEGF plus PDT combination therapy than with PDT monotherapy.

Data for CRT after 12 months of treatment, stratified by disease type, are shown in Figure 2D. No notable difference between disease type was observed; in both groups, the mean CRT after 12 months was approximately $220 \mu \mathrm{m}$.

Data for CRT after 12 months of treatment, stratified by disease type and treatment modality, are shown in Figure 2E. Among the 13 typical AMD studies, anti-VEGF agents were the predominant treatment. Patients received either antiVEGF monotherapy (12 studies) or anti-VEGF plus PDT (1 study); there were no studies in which patients were treated with PDT monotherapy. Therefore, we considered that stratification of the typical AMD group would not provide meaningful data; as a result, only data for the PCV group were stratified and evaluated. Anti-VEGF monotherapy and anti-VEGF plus PDT combination therapy resulted in lower mean CRT (ie, an improvement) after 12 months compared with PDT monotherapy.

Data for the number of anti-VEGF injections during the first 12 months of treatment, stratified by disease type, are shown in Figure 2F. The mean number of anti-VEGF injections during the first 12 months of treatment tended to be lower for patients with PCV (4.6) than those with typical AMD (5.6).

Corresponding data stratified by both disease type and treatment modality are shown in S2 Figure and Figure 2G. For patients with PCV, the mean number of anti-VEGF injections during the first 12 months of treatment was lower when receiving anti-VEGF plus PDT combination therapy (3.2) than anti-VEGF monotherapy (5.3).

When outcomes were calculated using only the treatment arms for which all outcome data were available (VA, CRT, and injection number), no differences were observed in the results (data not shown).

\section{Discussion}

In the decade since the introduction of anti-VEGF drugs for Japanese patients with wAMD, 94 studies including clinical effectiveness measures (VA, CRT, and injection number) have been published, totaling 147 individually evaluable treatment arms. Among Japanese patients with wAMD, more studies evaluated treatment for PCV than typical AMD. Previous studies have suggested that PCV occurs at a higher rate in Japanese/Asian individuals than Caucasians, ${ }^{13-15}$ and that PCV is a relevant problem in Japan; ${ }^{34}$ hence, there is likely to be clinical interest in evaluating treatments for AMD in Japanese populations. Overall, the most common treatments for AMD were anti-VEGF drugs and PDT, either as monotherapy or in combination, which is in line with current recommendations both in Japan and globally ${ }^{20,35-37}$ where anti-VEGF therapy is the current standard-of-care.

The production of high-quality systematic reviews can provide a strong evidence base to underpin clinical care. ${ }^{38}$ The use of this methodology to evaluate wAMD treatment in Japan allowed us to assess a large body of pooled evidence on clinical outcomes and observe whether effectiveness aligns with current practice recommendations. The pooled analysis of efficacy outcomes in patients with typical AMD indicated that anti-VEGF monotherapy yielded better results than PDT monotherapy in terms of VA after 12 months of treatment. However, baseline values varied between treatment groups because of the different studies that were included in our analyses. Both anti-VEGF monotherapy and anti-VEGF plus PDT combination therapy yielded better results in terms of VA and CRT for patients with PCV than PDT monotherapy after 12 months of treatment. Moreover, anti-VEGF plus PDT combination therapy required fewer 
A

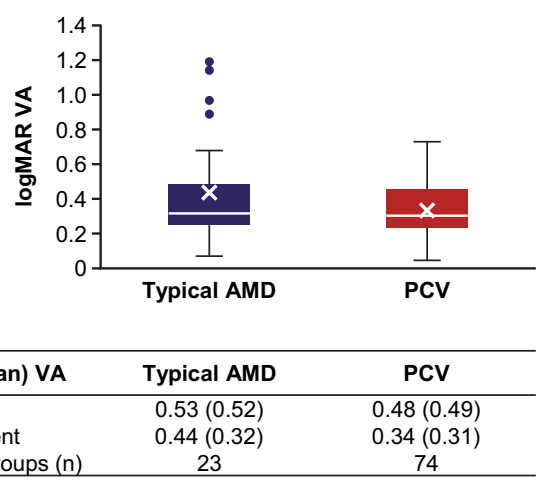

C

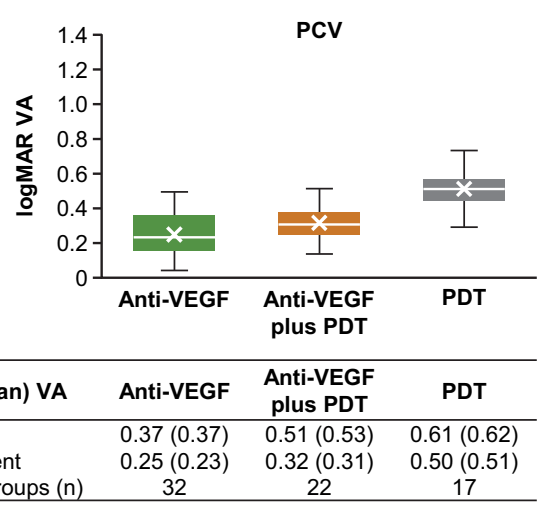

E

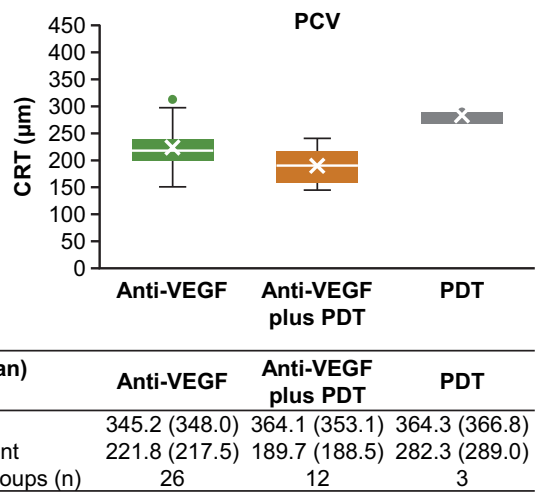

G

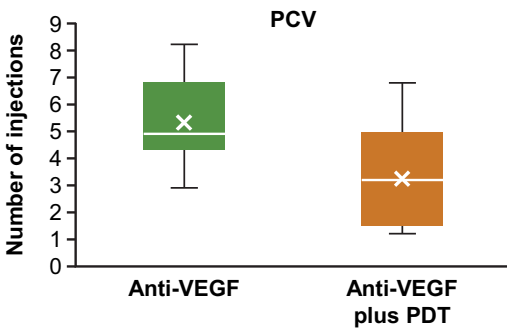

\begin{tabular}{lcc}
\hline $\begin{array}{l}\text { Mean (median) } \\
\text { number of injections }\end{array}$ & Anti-VEGF & $\begin{array}{c}\text { Anti-VEGF } \\
\text { plus PDT }\end{array}$ \\
\hline $\begin{array}{l}\text { During the first } 12 \\
\text { months of treatment }\end{array}$ & $5.3(4.9)$ & $3.2(3.2)$ \\
Treatment groups $(\mathrm{n})$ & 30 & 17 \\
\hline
\end{tabular}
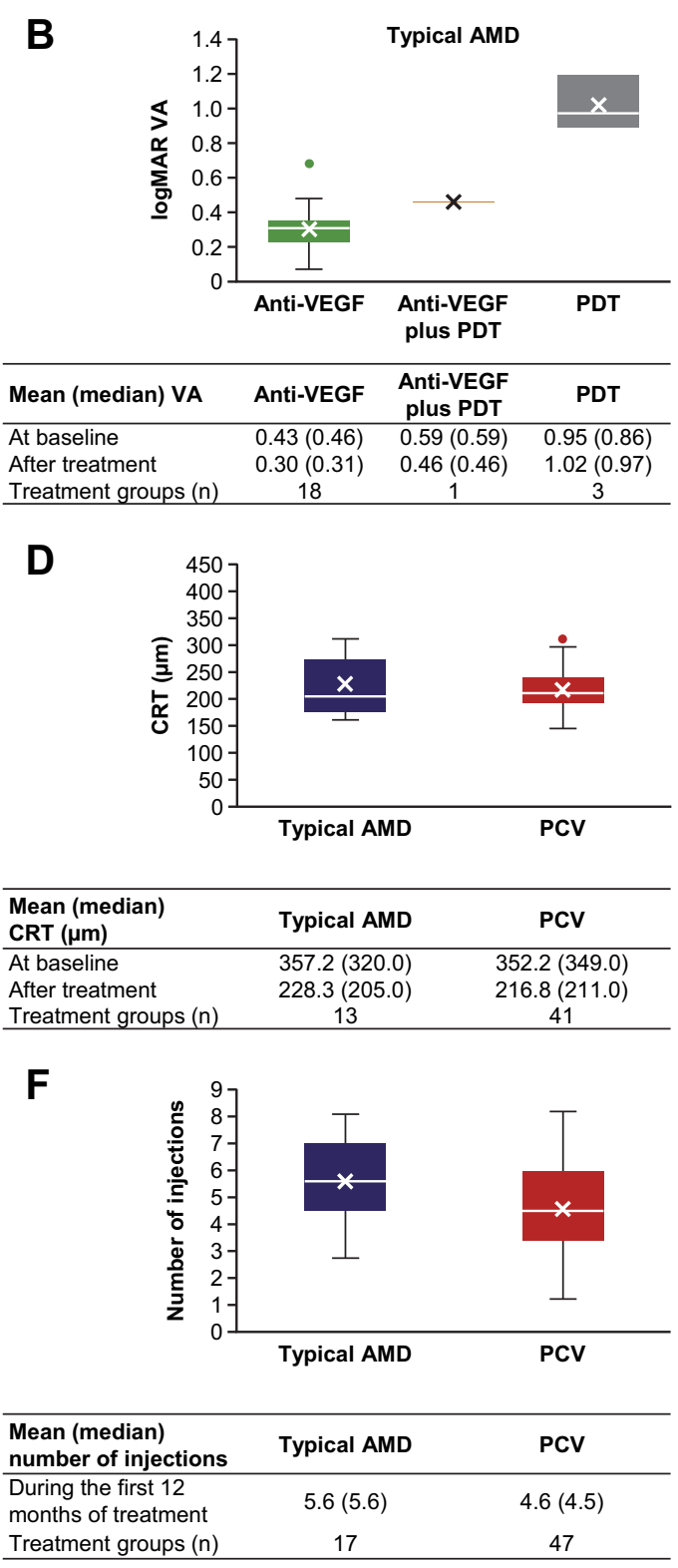

Figure 2 Outcomes after 12 months of treatment: (A) VA stratified by disease type; (B) VA stratified by disease type (typical AMD) and treatment modality; (C) VA stratified by disease type (PCV) and treatment modality; (D) CRT stratified by disease type; (E) CRT stratified by disease type (PCV) and treatment modality; (F) Number of anti-VEGF injections stratified by disease type; and (G) Number of anti-VEGF injections stratified by disease type (PCV) and treatment modality; baseline values vary by treatment group.

Abbreviations: AMD, age-related macular degeneration; CRT, central retinal thickness; logMAR, logarithm of the minimum angle of resolution; PCV, polypoidal choroidal vasculopathy; PDT, photodynamic therapy; VA, visual acuity; VEGF, vascular endothelial growth factor. 
injections than anti-VEGF monotherapy. The reduction in injection number is of particular interest to patients and clinicians. Intraocular injections require specialist expertise and can be labor-intensive and costly. ${ }^{39}$ Furthermore, many patients experience a psychosocial and practical burden from the treatment schedule. ${ }^{18,40,41}$ As a result, patients with wAMD in real-world practice are often undertreated, resulting in a lack of improvement (or even deterioration) in VA over time. ${ }^{42,43}$ Because of the highly invasive nature of treatment and the burdens associated with the treatment schedule, reduced dosing is desirable regardless of whether combination treatment or anti-VEGF monotherapy is used. As newer anti-VEGF agents are launched, ${ }^{44}$ it is hoped that treatments requiring fewer injections will become available.

It is clear from our study findings that progress has been made in the treatment of wAMD in the years since the introduction of anti-VEGF therapy in Japan. However, there remains a need for novel treatments and regimens that offer a better prognosis for VA while requiring fewer injections. ${ }^{39}$ Many recent studies have evaluated PRN, quarterly, and "treat and extend" dosing regimens instead of fixed dosing in an attempt to balance VA improvement and maintenance with fewer injections. ${ }^{45}$ The anti-VEGF antibody fragment brolucizumab, which is now approved in several countries including the United States and Japan, ${ }^{46}$ can be used with a 12 -weekly maintenance schedule. ${ }^{47}$ In addition to the possibility of treatment with fewer doses, brolucizumab has excellent fluid control. ${ }^{48}$ There is also interest in sustainedrelease delivery devices for both novel and approved agents, ${ }^{49-52}$ in oral anti-VEGF formulations, ${ }^{53,54}$ and in gene therapy. ${ }^{55,56}$ The diversity of subject areas covered in wAMD research within an elderly Japanese population was narrow, with some search terms returning little evidence. Treatment comparisons may have suffered from heterogeneity in the study design and procedures, and no statistical comparisons were conducted.

\section{Conclusions}

The findings outlined in this study represent a large body of evidence relating to wAMD treatment and treatment outcomes in Japan. During the decade since the introduction of anti-VEGF drugs in Japan, wAMD treatment has advanced dramatically. The present results appear to confirm the treatment outcomes observed in clinical practice and suggest that discrete patient populations may gain optimal benefits from differing management regimens. Nevertheless, the development of new drugs and treatment regimens that offer an improved prognosis for VA and require fewer injections remains an important unmet clinical need.

\section{Abbreviations}

AMD, age-related macular degeneration; CRT, central retinal thickness; IQR, interquartile range; logMAR, logarithm of the minimum angle of resolution; MeSH, medical subject heading; PCV, polypoidal choroidal vasculopathy; PDT, photodynamic therapy; PRISMA, Preferred Reporting Items for Systematic Reviews and Meta-Analyses; PRN, pro re nata; TIAB, title/abstract; VA, visual acuity; VEGF, vascular endothelial growth factor; wAMD, wet AMD.

\section{Data Sharing Statement}

All data supporting the results are included in the manuscript.

\section{Acknowledgments}

The authors would like to thank William YuanHao Kuan, of Syneos Health, Tokyo, Japan, for assistance with the first round of screening and data extraction. We also thank Sally-Anne Mitchell, $\mathrm{PhD}$, of Edanz, for providing medical writing assistance, which was funded by Novartis Pharma K.K. through EMC K.K. in accordance with Good Publication Practice (GPP3) guidelines (http://www.ismpp.org/gpp3). Some data from this systematic literature review were previously presented at the 59th Annual Meeting of the Japanese Retina and Vitreous Society, Fukuoka, Japan, November 2020.

\section{Funding}

This study was funded by Novartis Pharma K.K., who were involved in the study design, data collection and analysis, decision to publish, and preparation of the manuscript. 


\section{Disclosure}

KT reports receiving honoraria for advisory board participation from Novartis Pharma K.K. for the current work; and grants from Hoya Corporation, Otsuka Pharmaceutical Co., Ltd., and Santen Pharmaceutical Co., Ltd., consulting fees from Kyowa Kirin Co., Ltd. and Santen Pharmaceutical Co., Ltd., and honoraria for presentations from Bayer Yakuhin Ltd., Kyowa Kirin Co., Ltd., and Santen Pharmaceutical Co., Ltd., outside of the submitted work.

TI reports receiving honoraria for advisory board participation from Novartis Pharma K.K. for the current work; and grants from Alcon Japan Ltd., AMO Japan K.K., Bayer Yakuhin Ltd., Hoya Corporation, NIDEK Co., Ltd., Otsuka Pharmaceutical Co., Ltd., Pfizer Japan Inc., Santen Pharmaceutical Co., Ltd., Senju Pharmaceutical Co., Ltd., and Topcon Corporation, consulting fees from Bayer Yakuhin Ltd., Chugai Pharmaceutical Co., Ltd., and Nippon Boehringer Ingelheim Co., Ltd., honoraria for presentations from Alcon Japan Ltd., Bayer Yakuhin Ltd., Canon Inc., Chugai Pharmaceutical Co., Ltd., JFC Sales Plan Co., Ltd., Novartis Pharma K.K., NIDEK Co., Ltd., Otsuka Pharmaceutical Co., Ltd., Santen Pharmaceutical Co., Ltd., Senju Pharmaceutical Co., Ltd., and Topcon Corporation, payment for expert testimony from Nippon Boehringer Ingelheim Co., Ltd., and patent involvement with Topcon Corporation, outside of the submitted work.

SI reports receiving honoraria for advisory board participation from Novartis Pharma K.K. for the current work; and grants from AbbVie GK, Alcon Japan Ltd., Alpha Communication, AMO Japan K.K., Bayer Yakuhin Ltd., Bonac Corporation, Bloom Technology Corporation, NIDEK Co., Ltd., Otsuka Pharmaceutical Co., Ltd., Santen Pharmaceutical Co., Ltd., SEED Co., Ltd., Senju Pharmaceutical Co., Ltd., Wakamoto Pharmaceutical Co., Ltd., and Whitemedical Co., Ltd., honoraria for presentations from Bayer Yakuhin Ltd., Chugai Pharmaceutical Co., Ltd., Hoya Corporation, Kowa Company, Ltd., NIDEK Co., Ltd., Nitto Medic Co., Ltd., Novartis Pharma K.K., Santen Pharmaceutical Co., Ltd., Senju Pharmaceutical Co., Ltd., and Wakamoto Pharmaceutical Co., Ltd., honoraria for manuscript writing from SENTAN IGAKU-SHA Ltd., payment for expert testimony from Bloom Technology Corporation, Chugai Pharmaceutical Co., Ltd., Kyowa Kirin Co., Ltd., Mylan EPD G.K., and SENTAN IGAKU-SHA Ltd., travel support from Alcon Pharma, Bayer Yakuhin Ltd., Chugai Pharmaceutical Co., Ltd., Hoya Corporation, Kowa Company, Ltd., NIDEK Co., Ltd., Nitto Medic Co., Ltd., Novartis Pharma K.K., Otsuka Pharmaceutical Co., Ltd., Santen Pharmaceutical Co., Ltd., Senju Pharmaceutical Co., Ltd., SENTAN IGAKU-SHA Ltd., and Wakamoto Pharmaceutical Co., Ltd., and patent involvement with Bonac Corporation, Chugai Pharmaceutical Co., Ltd., Senju Pharmaceutical Co., Ltd., SEED Co., Ltd., and NIDEK Co., Ltd., outside of the submitted work. SI also reports the following leadership roles: Director of the Japanese Ophthalmological Society; Executive Director of the Japanese Retina and Vitreous Society; Executive Director of the Japanese Society of Ophthalmic Diabetology; Director of the Japanese Society for Ocular Circulation; Councilor of the Japanese Ocular Inflammation Society; Councilor of the Japan Society of Diabetic Complications; Councilor of the Japanese Society of Anti-Aging Medicine; Councilor of the Japanese Society of Inflammation and Regeneration; Director of the Japanese Society for Ocular Pharmacology; Director of the Japanese Society for Microcirculation; International Commissioner of the Macula Society; and Vice Chairman of the Global Ocular Inflammation Workshops.

AM, RT, ST, and KI are employees of Novartis Pharma K.K. The authors report no other conflicts of interest in this work.

\section{References}

1. Pascolini D, Mariotti SP. Global estimates of visual impairment: 2010. Br J Ophthalmol. 2012;96(5):614-618. doi:10.1136/bjophthalmol-2011300539

2. Taylor HR, Keeffe JE. World blindness: a 21st century perspective. Br J Ophthalmol. 2001;85(3):261-266. doi:10.1136/bjo.85.3.261

3. Ferris FL 3rd, Wilkinson CP, Bird A, et al. Clinical classification of age-related macular degeneration. Ophthalmology. 2013;120(4):844-851. doi:10.1016/j.ophtha.2012.10.036

4. Wong WL, Su X, Li X, et al. Global prevalence of age-related macular degeneration and disease burden projection for 2020 and 2040: a systematic review and meta-analysis. Lancet Glob Health. 2014;2(2):e106-116. doi:10.1016/S2214-109X(13)70145-1

5. Jonas JB, Cheung CMG, Panda-Jonas S. Updates on the epidemiology of age-related macular degeneration. Asia Pac J Ophthalmol. 2017;6 (6):493-497. doi:10.22608/APO.2017251

6. Obata R, Yanagi Y, Inoue T, et al. Prevalence and factors associated with age-related macular degeneration in a southwestern island population of Japan: the Kumejima Study. Br J Ophthalmol. 2018;102(8):1047-1053. doi:10.1136/bjophthalmol-2016-309980

7. Nakata I, Yamashiro K, Nakanishi H, et al. Prevalence of AMD in the Japanese: the Nagahama study. Investig Ophthalmol Vis Sci. $2012 ; 53: 3807$. 
8. Nakata I, Yamashiro K, Nakanishi H, et al. Prevalence and characteristics of age-related macular degeneration in the Japanese population: the Nagahama study. Am J Ophthalmol. 2013;156(5):1002-1009. doi:10.1016/j.ajo.2013.06.007

9. Kume A, Ohshiro T, Sakurada Y, Kikushima W, Yoneyama S, Kashiwagi K. Treatment patterns and health care costs for age-related macular degeneration in Japan: an analysis of National Insurance claims data. Ophthalmology. 2016;123(6):1263-1268. doi:10.1016/j.ophtha.2016.01.042

10. Ferris FL 3rd, Fine SL, Hyman L. Age-related macular degeneration and blindness due to neovascular maculopathy. Arch Ophthalmol. 1984;102 (11):1640-1642. doi:10.1001/archopht.1984.01040031330019

11. Lipecz A, Miller L, Kovacs I, et al. Microvascular contributions to age-related macular degeneration (AMD): from mechanisms of choriocapillaris aging to novel interventions. Geroscience. 2019;41(6):813-845. doi:10.1007/s11357-019-00138-3

12. Yasuda M, Kiyohara Y, Hata Y, et al. Nine-year incidence and risk factors for age-related macular degeneration in a defined Japanese population: the Hisayama study. Ophthalmology. 2009;116(11):2135-2140. doi:10.1016/j.ophtha.2009.04.017

13. Fujiwara K, Yasuda M, Hata J, et al. Prevalence and risk factors for polypoidal choroidal vasculopathy in a general Japanese population: the Hisayama study. Semin Ophthalmol. 2018;33(6):813-819. doi:10.1080/08820538.2018.1506483

14. Lorentzen T, Subhi Y, Sørensen TL. Prevalence of polypoidal choroidal vasculopathy in white patients with exudative age-related macular degeneration: systematic review and meta-analysis. Retina. 2018;38(12):2363-2371. doi:10.1097/IAE.0000000000001872

15. Yanagi Y, Foo VHX, Yoshida A. Asian age-related macular degeneration: from basic science research perspective. Eye. 2019;33(1):34-49. doi:10.1038/s41433-018-0225-x

16. Mitchell J, Bradley C. Quality of life in age-related macular degeneration: a review of the literature. Health Qual Life Outcomes. $2006 ; 4(1): 97$. doi:10.1186/1477-7525-4-97

17. Yuzawa M, Fujita K, Tanaka E, Wang EC. Assessing quality of life in the treatment of patients with age-related macular degeneration: clinical research findings and recommendations for clinical practice. Clin Ophthalmol. 2013;7:1325-1332. doi:10.2147/OPTH.S45248

18. Honda S, Yanagi Y, Koizumi H, et al. Impact of neovascular age-related macular degeneration: burden of patients receiving therapies in Japan. Sci Rep. 2021;11(1):13152. doi:10.1038/s41598-021-92567-4

19. Roberts CB, Hiratsuka Y, Yamada M, et al. Economic cost of visual impairment in Japan. Arch Ophthalmol. 2010;128(6):766-771. doi:10.1001/ archophthalmol.2010.86

20. Takahashi K, Ogura Y, Ishibashi T, Shiraga F, Yuzawa M. Treatment guidelines for age-related macular degeneration. J Jpn Ophthalmol Soc. 2012;116(12):1150-1155. [in Japanese]

21. Yorston D. Anti-VEGF drugs in the prevention of blindness. Community Eye Health. 2014;27(87):44-46.

22. Report on the deliberation results. Ministry of Health, Labour and Welfare. Available from: https://www.pmda.go.jp/files/000153104.pdf. Accessed June 15, 2021.

23. Bakri SJ, Thorne JE, Ho AC, et al. Safety and efficacy of anti-vascular endothelial growth factor therapies for neovascular age-related macular degeneration: a report by the American Academy of Ophthalmology. Ophthalmology. 2019;126(1):55-63. doi:10.1016/j.ophtha.2018.07.028

24. Solomon SD, Lindsley K, Vedula SS, Krzystolik MG, Hawkins BS. Anti-vascular endothelial growth factor for neovascular age-related macular degeneration. Cochrane Database Syst Rev. 2014;8(8):CD005139. doi:10.1002/14651858.CD005139.pub3

25. Rosenfeld PJ, Brown DM, Heier JS, et al. Ranibizumab for neovascular age-related macular degeneration. N Engl J Med. 2006;355(14):1419-1431. doi:10.1056/NEJMoa054481

26. Schmidt-Erfurth U, Kaiser PK, Korobelnik JF, et al. Intravitreal aflibercept injection for neovascular age-related macular degeneration: ninety-sixweek results of the VIEW studies. Ophthalmology. 2014;121(1):193-201. doi:10.1016/j.ophtha.2013.08.011

27. Ogura Y, Terasaki H, Gomi F, et al. Efficacy and safety of intravitreal aflibercept injection in wet age-related macular degeneration: outcomes in the Japanese subgroup of the VIEW 2 study. Br J Ophthalmol. 2015;99(1):92-97. doi:10.1136/bjophthalmol-2014-305076

28. Ishikawa M, Jin D, Sawada Y, Abe S, Yoshitomi T. Future therapies of wet age-related macular degeneration. J Ophthalmol. 2015;2015:138070. doi:10.1155/2015/138070

29. Papadopoulos Z. Recent developments in the treatment of wet age-related macular degeneration. Curr Med Sci. 2020;40(5):851-857. doi:10.1007/ s11596-020-2253-6

30. Ammar MJ, Hsu J, Chiang A, Ho AC, Regillo CD. Age-related macular degeneration therapy: a review. Curr Opin Ophthalmol. 2020;31 (3):215-221. doi:10.1097/ICU.0000000000000657

31. Moher D, Liberati A, Tetzlaff J, Altman DG, The PRISMA Group. Preferred reporting items for systematic reviews and meta-analyses: the PRISMA statement. Ann Intern Med. 2009;151(4):264-269, W64. doi:10.7326/0003-4819-151-4-200908180-00135

32. Dias S, Welton NJ, Sutton AJ, Ades AE. NICE DSU technical support document 1: Introduction to evidence synthesis for decision making. National Institute for Health and Clinical Excellence. Available from: https://www.ncbi.nlm.nih.gov/books/NBK395870/pdf/Bookshelf NBK395870.pdf. Accessed June 15, 2021.

33. Takahashi K, Ishibashi T, Ogura Y, Yuzawa M. Working group for establishing diagnostic criteria for age-related macular degeneration. Classification and diagnostic criteria of age-related macular degeneration. J Jpn Ophthalmol Soc. 2008;112(12):1076-1084. [in Japanese]

34. Tsujikawa A, Akagi-Kurashige Y, Yuzawa M, et al. Baseline data from a multicenter, 5-year, prospective cohort study of Japanese age-related macular degeneration: an AMD2000 report. Jpn J Ophthalmol. 2018;62(2):127-136. doi:10.1007/s10384-017-0556-3

35. Flaxel CJ, Adelman RA, Vemulakonda A, et al. Age-related macular degeneration preferred practice pattern. Am Acad Ophthalmol. 2020;127(1): $\mathrm{P} 1-65$.

36. Age-related macular degeneration NICE guideline [NG82]. National Institute for Health and Care Excellence. Available from: https://www.nice. org.uk/guidance/ng82/chapter/recommendations. Accessed June 15, 2021.

37. 2019 Clinical Practice Guide for the diagnosis, treatment and management of age-related macular degeneration. Optometry Australia. Available from: https://www.optometry.org.au/wp-content/uploads/Professional_support/Practice_notes/AMD-Clinical-Practice-Guide-2019_final_designed_ v5.pdf. Accessed June 15, 2021

38. Lindsley K, Li T, Ssemanda E, Virgili G, Dickersin K. Interventions for age-related macular degeneration: are practice guidelines based on systematic reviews? Ophthalmology. 2016;123(4):884-897. doi:10.1016/j.ophtha.2015.12.004

39. Baumal CR. Wet age-related macular degeneration: treatment advances to reduce the injection burden. Am J Manag Care. 2020;26(5 Suppl):S103S111. 
40. Boyle J, Vukicevic M, Koklanis K, Itsiopoulos C, Rees G. Experiences of patients undergoing repeated intravitreal anti-vascular endothelial growth factor injections for neovascular age-related macular degeneration. Psychol Health Med. 2018;23(2):127-140. doi:10.1080/ 13548506.2016.1274040

41. Thier A, Holmberg C. The patients' view: age-related macular degeneration and its effects - a meta-synthesis. Disabil Rehabil. 2020;1-11. doi:10.1080/09638288.2020.1775901

42. Holz FG, Tadayoni R, Beatty S, et al. Multi-country real-life experience of anti-vascular endothelial growth factor therapy for wet age-related macular degeneration. Br J Ophthalmol. 2015;99(2):220-226. doi:10.1136/bjophthalmol-2014-305327

43. Ciulla TA, Hussain RM, Pollack JS, Williams DF. Visual acuity outcomes and anti-vascular endothelial growth factor therapy intensity in neovascular age-related macular degeneration patients: a real-world analysis of 49485 eyes. Ophthalmol Retina. 2020;4(1):19-30. doi:10.1016/ j.oret.2019.05.017

44. Pugazhendhi A, Hubbell M, Jairam P, Ambati B. Neovascular macular degeneration: a review of etiology, risk factors, and recent advances in research and therapy. Int J Mol Sci. 2021;22(3):1170. doi:10.3390/ijms22031170

45. Khanna S, Komati R, Eichenbaum DA, Hariprasad I, Ciulla TA, Hariprasad SM. Current and upcoming anti-VEGF therapies and dosing strategies for the treatment of neovascular AMD: a comparative review. BMJ Open Ophthalmol. 2019;4(1):e000398. doi:10.1136/bmjophth-2019-000398

46. Markham A. Brolucizumab: first approval. Drugs. 2019;79(18):1997-2000. doi:10.1007/s40265-019-01231-9

47. BEOVU (brolucizumab-dbll) injection, for intravitreal use [Prescribing Information]. East Hanover, NJ, USA: Novartis Pharmaceuticals Corp.; 2020. Available from: https://www.novartis.us/sites/www.novartis.us/files/beovu.pdf. Accessed February 8, 2022.

48. Nguyen QD, Das A, Do DV, et al. Brolucizumab: evolution through preclinical and clinical studies and the implications for the management of neovascular age-related macular degeneration. Ophthalmology. 2020;127(7):963-976. doi:10.1016/j.ophtha.2019.12.031

49. Chittasupho C, Kengtrong K, Chalermnithiwong S, Sarisuta N. Anti-angiogenesis by dual action of R5K peptide conjugated itraconazole nanoparticles. AAPS PharmSciTech. 2020;21(3):74. doi:10.1208/s12249-019-1568-8

50. Bhatt P, Narvekar P, Lalani R, Chougule MB, Pathak Y, Sutariya V. An in vitro assessment of thermo-reversible gel formulation containing sunitinib nanoparticles for neovascular age-related macular degeneration. AAPS PharmSciTech. 2019;20(7):281. doi:10.1208/s12249-019-1474-0

51. Liu W, Borrell MA, Venerus DC, Mieler WF, Kang-Mieler JJ. Characterization of biodegradable microsphere-hydrogel ocular drug delivery system for controlled and extended release of ranibizumab. Transl Vis Sci Technol. 2019;8(1):12. doi:10.1167/tvst.8.1.12

52. Lim JI, Niec M, Wong V. One year results of a Phase 1 study of the safety and tolerability of combination therapy using sustained release intravitreal triamcinolone acetonide and ranibizumab for subfoveal neovascular AMD. Br J Ophthalmol. 2015;99(5):618-623. doi:10.1136/ bjophthalmol-2014-306002

53. Cohen MN, O'Shaughnessy D, Fisher K, et al. APEX: a Phase II randomised clinical trial evaluating the safety and preliminary efficacy of oral X-82 to treat exudative age-related macular degeneration. Br J Ophthalmol. 2021;105(5):716-722. doi:10.1136/bjophthalmol-2020-316511

54. Tarallo V, Iaccarino E, Cicatiello V, Sanna R, Ruvo M, De Falco S. Oral delivery of a tetrameric tripeptide inhibitor of VEGFR1 suppresses pathological choroid neovascularization. Int J Mol Sci. 2020;21(2):410. doi:10.3390/ijms21020410

55. Guimaraes TAC, Georgiou M, Bainbridge JWB, Michaelides M. Gene therapy for neovascular age-related macular degeneration: rationale, clinical trials and future directions. Br J Ophthalmol. 2021;105(2):151-157. doi:10.1136/bjophthalmol-2020-316195

56. Reid CA, Nettesheim ER, Connor TB, Lipinski DM. Development of an inducible anti-VEGF rAAV gene therapy strategy for the treatment of wet AMD. Sci Rep. 2018;8(1):11763. doi:10.1038/s41598-018-29726-7

\section{Publish your work in this journal}

Clinical Ophthalmology is an international, peer-reviewed journal covering all subspecialties within ophthalmology. Key topics include: Optometry; Visual science; Pharmacology and drug therapy in eye diseases; Basic Sciences; Primary and Secondary eye care; Patient Safety and Quality of Care Improvements. This journal is indexed on PubMed Central and CAS, and is the official journal of The Society of Clinical Ophthalmology (SCO). The manuscript management system is completely online and includes a very quick and fair peer-review system, which is all easy to use. Visit http://www. dovepress.com/testimonials.php to read real quotes from published authors.

Submit your manuscript here: https://www.dovepress.com/clinical-ophthalmology-journal 